\title{
A rare pediatric case of neurobrucellosis with bilateral optic neuritis
}

\author{
Cengiz Havalı ${ }^{1 \oplus}$, Eren Çağan²ْ \\ Divisions of ${ }^{1}$ Pediatric Neurology and ${ }^{2}$ Pediatric Infectious Diseases, Department of Pediatrics, Bursa Yüksek Ihtisas Research and \\ Training Hospital, Bursa, Turkey.
}

\begin{abstract}
Background. The estimated incidence of central nervous system involvement in brucellosis ranges between 0 and $17.8 \%$. Optic neuritis is infrequently seen in the clinical presentation of neurobrucellosis. Only six cases of neurobrucellosis manifesting with optic neuritis have been previously reported in the literature during childhood. Moreover, four of these were isolated optic neuritis.

Case. An 11-year-old boy presented with the complaint of bilateral visual loss which was more prominent in the left eye than the right. He was diagnosed with brucellosis two months prior. His fundus examination revealed mild papilledema of the right eye and remarkable papilledema of the left eye. Brucella agglutination titer of serum was 1/640. Cerebrospinal fluid (CSF) cultures were negative, but polymerase chain reaction (PCR) examination in CSF was positive for Brucella melitensis. Antibiotic and pulse methylprednisolone treatments were administered. The visual acuity returned incompletely within the 12-month follow-up period.
\end{abstract}

Conclusion. Isolated optic neuritis is a rare manifestation of neurobrucellosis in children.

Key words: optic neuritis, neurobrucellosis, children.

Brucellosis is a common zoonotic disease that is seen worldwide and mainly raises a public health problem in Mediterranean countries, including Turkey., ${ }^{1,2}$ It has been considered a notable illness, particularly in endemic areas of Turkey. The number of cases decreased from 14644 (morbidity rate, 20.32/100 000) in 2005 to 4173 (morbidity rate, 5.30/100 000) in 2015 in Turkey. However, after 2015 the morbidity rate has revived and reached 7.99 in 2017..$^{3}$ All age groups and both genders are affected. In endemic areas, children represent $20-25 \%$ of cases. ${ }^{2}$ It is a multisystem disease that mostly affects the hematopoietic, musculoskeletal, genitourinary, cardiovascular, respiratory, and central nervous systems. The incidence of central nervous system involvement in

\section{Cengiz Havalı}

cengizhavali@gmail.com

Received 4th May 2020, revised 29th June 2020,

5th July 2020, 16th July 2020, 23rd July 2020, 24th July 2020, accepted 5th August 2020. brucellosis ranges between 0 and $17.8 \%$ in various reports. Neurologic involvement of Brucella are infrequently encountered in childhood; the proportion of neurologic system involvement is $0.8 \%$ of children with systemic brucellosis. ${ }^{4}$ Neurologic system involvement presents as either a component of the current systemic disease or can be isolated. Clinical presentation of neurobrucellosis consists of meningitis, meningoencephalitis, meningovascular involvement, peripheral neuropathy, radiculopathy, cranial nerve involvement, and behavioural abnormalities. Meningitis and meningoencephalitis have been the most frequent presentation in clinical series. ${ }^{5}$

The rate of ocular involvement was found to be $3.35 \%$ in patients with brucellosis. Uveitis was the most frequent ocular presentation. The less common manifestations of ocular involvement were keratoconjunctivitis, corneal ulcers, iridocyclitis, choroiditis, optic neuritis, papilledema, and endophthalmitis. ${ }^{6}$ 
Optic neuritis presents considerably less commonly in children than adults, and most cases are due to an immune-mediated process following an antecedent viral illness. Postinfectious, post-vaccination inflammation, Leber's hereditary optic neuropathy (LHON), and infectious causes (Bartonella, syphilis, Lyme, Toxocara, and toxoplasmosis can damage the optic nerve.). Optic neuritis has also been due to acute infection with Epstein Barr Virus (EBV), Borrelia, measles, mumps and varicella. In one series including patients with optic neuritis of all ages, children comprised 5\% of cases. Optic neuritis may be associated with infections of sinuses or orbital components and infiltrative or infectious diseases of the brain and meninges that involve the optic nerves. ${ }^{7}$ Optic neuritis may occasionally be the first manifestation of a demyelinating disease such as multiple sclerosis. ${ }^{8}$ Optic neuritis related to Brucella infection is scarce and rarely reported in the literature.

One patient who suffered from optic neuritis in the course of systemic brucellosis is presented.

\section{Case Report}

An 11-year-old boy presented with the complaint of bilateral visual loss which was more prominent in the left eye than the right. The child was the sixth child of the family, with no remarkable prenatal or neonatal event and with healthy psychomotor development. He had been diagnosed with brucellosis with fever, arthralgia, and weakness two months prior. The diagnosis had been confirmed by positive serologic tests. The patient's parents had also suffered the same findings in the last year and had been diagnosed with brucellosis.

Moreover, the family livesin a rural area, in which brucellosis is endemic and usually consumes unpasteurized dairy products, especially soft cheese. Oral antibiotic treatment had been prescribed, consisting of co-trimoxazole and rifampicin for six weeks. Although his constitutional symptoms had regressed, he had gradually decreasing visual acuity for the last ten days. He had no vaccination nor infection history in the previous month.

A detailed ophthalmic examination of the patient was performed. Best-corrected visual acuity (BCVA) was counting from one-meter on the right eye, and hand motion on the left eye. Direct light reflexes were weak in both eyes. No relative afferent pupillary defect was detected with biomicroscopic slit-lamp examination. Intraocular pressure was measured normally by Goldmann applanation tonometer. Fundus examination revealed mild optic disc swelling on the right eye, and remarkable optic disc swelling on the left eye. Visual field test could not be performed because the patient did not cooperate with the test. Other systemic and neurologic examinations were all normal.

Blood cell counts, liver function tests, serum electrolytes, and erythrocyte sedimentation rate were within normal limits. Antinuclear antibodies, serum antibodies for Ebstein Bar Virus (EBV), cytomegalovirus and toxoplasma gondii were unremarkable. Brucella agglutination titer of serum was 1/640. Lumbar puncture revealed clear cerebrospinal fluid (CSF) with normal opening pressure which was $80 \mathrm{~mm} / \mathrm{H} 2 \mathrm{O}$ (normal range, $60-200 \mathrm{~mm} /$ $\mathrm{H} 2 \mathrm{O}$ ) and white blood cell count of three cells/ $\mathrm{mL}$, glucose $45 \mathrm{mg} / \mathrm{dl}$ (normal range, 40-70 $\mathrm{mg} / \mathrm{dl}$ ), and protein $65 \mathrm{mg} / \mathrm{dl}$ (normal range, $15-45 \mathrm{mg} / \mathrm{dL}$ ). Unfortunately, we did not find simultaneous blood glucose levels because the physicians who performed the lumbar puncture, failed to evaluate the concurrent blood glucose level. CSF cultures were negative for four weeks, but polymerase chain reaction (PCR) examination in CSF was positive for Brucella melitensis.

His contrast-enhanced brain magnetic resonance imaging (MRI) had no pathologic findings. Visual evoked potential testing (VEPs) showed a late response with low amplitude in both eyes. The right and left P100 latencies were $130 \mathrm{~ms}$ (reference range $80-120 \mathrm{~ms}$ ) and $145 \mathrm{~ms}$, respectively. The auditory brain stem response study revealed normal responses. 
Antibiotic therapy with doxycycline $(6 \mathrm{mg} /$ $\mathrm{kg} /$ day) and rifampicin (20 mg/kg/day) was administered for six months and ceftriaxone $100 \mathrm{mg} / \mathrm{kg} / \mathrm{d}$ for the first three weeks. Pulse methylprednisolone treatment $(30 \mathrm{mg} / \mathrm{kg} /$ day), was initiated for three days and continued (1 $\mathrm{mg} / \mathrm{kg} /$ day) throughout eleven days. Partial reversal of visual acuity was observed within the 12-month follow-up period. BCVA was counting from three meters on the right eye and, 0.15 with the Snellen chart on the left eye at the last ophthalmologic examination. Papilledema improved; however, both discs were pale, which is a sign of optic atrophy. Brucella agglutination titer of serum was 1/320 for immunoglobulin $G$ type antibodies and negative for immunoglobulin $\mathrm{M}$ type antibodies at the 12-month follow-up period.

Written informed consent was received from his parents. Parents were fully informed.

\section{Discussion}

Neurobrucellosis usually emerges with nonspecific manifestations and may imitate various pathologies. Symptoms and signs can arise at the acute or chronic period, but neurologic symptoms are rarely the initial complaint. The history of ingesting milk or dairy products, lack of typical signs of other neurological diseases, and amelioration of symptoms with appropriate treatment in a clinical history are clues for physicians. ${ }^{2,5}$

Optic neuritis and other cranial nerve involvement may be part of the neurologic participation of brucellosis, such as meningitis or meningoencephalitis. Documented cranial nerve involvement of brucellosis includes vestibulocochlear, trigeminal, facial, abducens, oculomotor, and optic nerves. Any cranial nerve may involve isolated, or two and more nerves may participate in neurobrucellosis. ${ }^{9,10}$

Optic neuritis is very rare in children with neurobrucellosis. To date, six case reports have been reported. Four patients had isolated optic neuritis. ${ }^{11,12,14,15}$ Two patients also had other cranial nerve involvement along with the optic nerve. ${ }^{10,13}$ It has been denoted that antibiotic and prednisolone therapy was given to the patients in these reports. While optic neuritis had regressed in some of the patients, the rest of the patients had recovered with a sequel of vision impairment.

Our patient had been diagnosed with brucellosis in the last few months. Fever and arthralgia had recovered with antibiotic therapy for six weeks. However, vision loss developed after a fortnight of the end of the treatment. Acute visual impairment along with the history of diagnosis with brucellosis, ingesting unpasteurized milk products, living in the village and endemic area for brucellosis, lack of typical signs of a known other neurological disease, and a serum agglutination titer higher than 1:640 were essential data for suspicion of brucellosis. Moreover, the diagnosis was confirmed with PCR examination of CSF. Probable other causes of optic neuritis were distinguished with studies for autoimmune diseases, viral and parasite agents. The development of optic neuritis despite the treatment of brucellosis for six weeks was associated with the failure of the therapy.

A diagnosis of neurobrucellosis can be established by the isolation of Brucella species from blood, bone marrow or CSF. Serum and CSF titer higher than 1:160 and 1:80, respectively, abnormal CSF findings with increased protein and lymphocytic pleocytosis reinforce the diagnosis in the laboratory. ${ }^{5}$ Recently, it has been issued that PCR assay could be useful as a sensitive and accurate diagnostic tool as well as blood culture. ${ }^{1}$ In our patient, Brucella was verified with PCR assay.

The treatment of central nervous system complications of brucellosis poses a particular problem because of the need to achieve high concentrations of drugs in the CSF. Recently, it has been reported that ceftriaxone-based regimens provided significantly more therapeutic success than the oral regimen and argued that the achievement of ceftriaxone 
is due to its high rate of free diffusion to body fluids. ${ }^{16}$ Ciprofloxacin, combined with doxycycline and rifampicin, were found to be useful in the treatment of neurobrucellosis. ${ }^{17}$ Doxycycline, rifampicin and ceftriaxone were given to our patient. Ciprofloxacin was not chosen due to its severe side effects in children.

In the differential diagnosis of optic neuritis; probable infectious causes should be investigated. A CSF glucose concentration less than $40 \mathrm{mg} / \mathrm{dL}$ or a ratio of less than twothirds of the plasma glucose level is considered abnormal. Very low CSF glucose values mostly occur in acute bacterial meningitis, but mildly to moderately low values are commonly seen in fungal, parasitic, protozoal, spirochetal, and tuberculous meningitis. A low CSF glucose level is less common in viral meningitis but can also appear with noninfectious conditions, including plasma hypoglycemia, meningeal carcinomatosis, sarcoidosis, chemical meningitis, and subarachnoid haemorrhage. ${ }^{18}$ We had no simultaneous CSF glucose but increased CSF protein value. Since, we have already no doubt about the diagnosis of neurobrucellosis in that period with the patient's history, clinical findings, and laboratory results, we thought that the lack of concurrent blood glucose level was acceptable. AntiAquaporin-4 and anti myelin oligodendrocyte glycoprotein antibodies can be studied for Neuromyelitis Optica in the presence of clinical suspicion and the relevant imaging findings. Otherwise, increased intracranial pressure due to neurobrucellosis must be kept in mind. Such patients also may come to remedy with similar symptoms and findings of optic neuritis. ${ }^{19,20}$ Therefore, to determine CSF, pressure by lumbar puncture is the rule for definitive diagnosis. Also, LHON should be kept in mind in a patient with vision loss. However, LHON usually presents with subacute visual failure, which develops in several months in young adults. Besides, other neurologic abnormalities such as postural tremor, peripheral neuropathy, myopathy, or movement disorders can be associated with LHON. ${ }^{21}$
Isolated optic neuritis as a manifestation of neurobrucellosis is very rare in children. To date, four case reports have been issued. In this respect, when a patient presents with optic neuritis for a differential diagnosis a thorough evaluation is critical to differentiate viral pathogens from bacterial pathogens since the therapy needs necessary antibiotics.

\section{Acknowledgement}

The authors declare no conflict of interest. We are grateful to our patient and his family for their contributions to this study. Also, the authors are thankful to all laboratory workers for their vigorous efforts.

\section{REFERENCES}

1. Hekmatimoghaddam S, Sadeh M, Khalili MB, Mollaabedin M, Sazmand A. Comparison of PCR, Wright agglutination test and blood culture for diagnosis of brucellosis in suspected patients. Pak J Biol Sci 2013; 16: 1589-1592.

2. Mantur BG, Akki AS, Mangalgi SS, Patil SV, Gobbur RH, Peerapur BV. Childhood brucellosis--a microbiological, epidemiological and clinical study. J Trop Pediatr 2004; 50: 153-157.

3. TC. Sağlık Bakanlığı Istatistikler/Temel Sağlık Hizmetleri Genel Müdürlüğü Çalışma Yıllığı. (Available from https://hsgm.saglik.gov.tr/tr/ zoonotikvektorel-bruselloz/istatistik) (Cited 7 July 2020).

4. Lubani MM, Dudin KI, Araj GF, Manandhar DS, Rashid FY. Neurobrucellosis in children. Pediatr Infect Dis J 1989; 8: 79-82.

5. Yetkin MA, Bulut C, Erdinc FS, Oral B, Tulek $\mathrm{N}$. Evaluation of the clinical presentations in neurobrucellosis. Int J Infect Dis 2006; 10: 446-452.

6. Rolando I, Olarte L, Vilchez G, et al. Ocular manifestations associated with brucellosis: a 26-year experience in Peru. Clin Infect Dis 2008; 46: 13381345.

7. Yeh EA, Graves JS, Benson LA, Wassmer E, Waldman A. Pediatric optic neuritis. Neurology 2016; 87(9 Suppl 2): S53-S58.

8. Dell'Avvento S, Sotgiu MA, Manca S, Sotgiu G, Sotgiu S. Epidemiology of multiple sclerosis in the pediatric population of Sardinia, Italy. Eur J Pediatr 2016; 175: 19-29. 
9. Karakurum Goksel B, Yerdelen D, Karatas M, et al. Abducens nerve palsy and optic neuritis as initial manifestation in brucellosis. Scand J Infect Dis 2006; 38: 721-725.

10. Tali ET, Keskin T, Oznur, II, Simonson T, Yuh WT. MRI of brucella polyneuritis in a child. Neuroradiology 1996; 38(Suppl 1): S190-S192.

11. Karapinar B, Yilmaz D, Vardar F, Demircioglu O, Aydinok Y. Unusual presentation of brucellosis in a child: acute blindness. Acta Paediatr 2005; 94: 378380 .

12. Marques R, Martins C, Machado I, Monteiro JP, Campos N, Calhau P. Unilateral optic neuritis as a presentation of neurobrucellosis. Pediatr Rep 2011; 3: e11.

13. Tonekaboni SH, Karimi A, Armin S, Khase LA, Sabertehrani AS. Neurobrucellosis: a partially treatable cause of vision loss. Pediatr Neurol 2009; 40: 401-403.

14. Cavallaro N, Randone A, La Rosa L, Mughinin L. Bilateral papilledema in a patient with brucellosis. Metab Pediatr Syst Ophthalmol (1985). 1990; 13: 115118.

15. Abd Elrazak M. Brucella optic neuritis. Arch Intern Med 1991; 151: 776-778.
16. Erdem H, Ulu-Kilic A, Kilic S, et al. Efficacy and tolerability of antibiotic combinations in neurobrucellosis: results of the Istanbul study. Antimicrob Agents Chemother 2012; 56: 1523-1528.

17. Bektaş O, Ozdemir H, Yılmaz A, et al. An unusual case of neurobrucellosis presenting as demyelination disorder. Turk J Pediatr 2013; 55: 210-213.

18. Chow E, Troy SB. The differential diagnosis of hypoglycorrhachia in adult patients. Am J Med Sci 2014; 348: 186-190.

19. Collinge JE, Sprunger DT. Update in pediatric optic neuritis. Curr Opin Ophthalmol 2013; 24: 448-452.

20. Sinopidis X, Kaleyias J, Mitropoulou K, Triga M, Kothare SV, Mantagos S. An uncommon case of pediatric neurobrucellosis associated with intracranial hypertension. Case Rep Infect Dis 2012; 2012: 492467.

21. Yu-Wai-Man P, Chinnery PF. Leber Hereditary Optic Neuropathy. In: Adam MP, Ardinger $\mathrm{HH}$ Pagon RA, Wallace SE, Bean LJH, Stephens K, Amemiya A, (eds). GeneReviews ${ }^{\circledR}$ [Internet]. Seattle (WA): University of Washington, Seattle; 1993-2020. PMID: 20301353. 